\title{
artigo
}

\section{Análise do programa de controle do tabagismo em um município de médio porte do Paraná}

\author{
Analysis of the smoking control program in a middle municipality in Paraná \\ Análisis del programa de control del humo en un municipio medio de Paraná
}

\begin{abstract}
RESUMO
Objetivo: Analisar os desfechos do tratamento para a cessação tabágica oferecido pelas Unidades Básicas de Saúde, inseridas no Programa de Controle do Tabagismo, de um município de médio porte do Estado do Paraná. Método: Pesquisa documental, descritiva, de abordagem quantitativa. Os dados utilizados incluíram os planos municipais e os documentos disponíveis na Secretaria Municipal de Saúde do município do período de 2015 a 2019. Os resultados foram tabulados no Software Microsoft Excel 2016, analisados por estatística descritiva e inferencial, aplicado o teste de qui-quadrado de Pearson com auxílio do ambiente estatístico Epilnfo versão 7.0. Resultados: Durante o período analisado, o município assistiu 2.339 usuários. Observou-se queda na procura e na efetividade do tratamento somado ao aumento nas taxas de abandono do tratamento. Conclusão: 0 município oferece atendimento às pessoas tabagistas, conforme previsto pelo Programa Nacional de Controle do Tabagismo, contudo, apresenta fragilidades para que o mesmo ocorra de forma exitosa.
\end{abstract}

DESCRITORES: Tabagismo; Atenção Primária à Saúde; Prevenção do Hábito de Fumar.

\section{ABSTRACT}

Objective: To analyze the treatment outcomes for smoking cessation offered by the Basic Health Units, inserted in the Tobacco Control Program, of a medium-sized municipality in the State of Paraná. Method: Documentary, descriptive research with a quantitative approach. The data used included the municipal plans and the documents available at the Municipal Health Department of the municipality from 2015 to 2019. The results were tabulated in the Microsoft Exel 2016 Software, analyzed by descriptive and inferential statistics, using the chi-square test of Pearson with the aid of the Epilnfo version 7.0 statistical environment. Results: During the period analyzed, the municipality assisted 2,339 users. There was a decrease in demand and treatment effectiveness, in addition to an increase in treatment dropout rates. Conclusion: The municipality offers assistance to smokers, as provided for by the National Tobacco Control Program, however, it presents weaknesses for it to occur successfully. DESCRIPTORS: Tobacco Use Disorder; Primary Health Care; Smoking Prevention.

\section{RESUMEN}

Objetivo: Analizar los resultados del tratamiento para el abandono del hábito de fumar que ofrecen las Unidades Básicas de Salud, insertas en el Programa de Control del Tabaco, de un municipio mediano del Estado de Paraná. Método: Investigación documental descriptiva con enfoque cuantitativo. Los datos utilizados incluyeron los planes municipales y los documentos disponibles en la Secretaría Municipal de Salud del municipio de 2015 a 2019. Los resultados se tabularon en el Software Microsoft Excel 2016, analizados mediante estadística descriptiva e inferencial, utilizando la prueba de chi-cuadrado de Pearson. con la ayuda del entorno estadístico Epilnfo versión 7.0. Resultados: Durante el período analizado, el municipio atendió a 2.339 usuarios. Hubo una disminución en la demanda y la efectividad del tratamiento, además de un aumento en las tasas de abandono del tratamiento. Conclusión: El municipio ofrece asistencia a los fumadores, conforme a lo previsto por el Programa Nacional de Control del Tabaco, sin embargo, presenta debilidades para que esto ocurra con éxito.

DESCRIPTORES: Tabaquismo; Atención Primaria de Salud; Prevención del Hábito de Fumar.

RECEBIDO EM: 17/02/2021 APROVADO EM: 17/03/2021

\section{Graziele Adrieli Rodrigues Pires}

Enfermeira. Mestranda pelo Programa de Pós-Graduação em Enfermagem da Universidade Estadual de Maringá (UEM). Maringá, Paraná, Brasil.

ORCID: 0000-0002-9673-9218 


\section{Patrícia Bossolani Charlo}

Enfermeira. Doutoranda pelo Programa de Pós-Graduação em Enfermagem da Universidade Estadual de Maringá (UEM). Maringá, Paraná, Brasil.

ORCID: 0000-0002-8262-2086

\section{Francielle Renata Danielli Martins Marques}

Enfermeira. Mestranda pelo Programa de Pós-Graduação em Enfermagem da Universidade Estadual de Maringá (UEM). Maringá, Paraná, Brasil.

ORCID: 0000-0002-8578-9615

\section{Eduardo Rocha Covre}

Enfermeiro. Doutorando pelo Programa de Pós-Graduação em Enfermagem da Universidade Estadual de Maringá (UEM). Maringá, Paraná, Brasil.

ORCID: 0000-0001-7338-121X

\section{Marcelle Paiano}

Doutora em Enfermagem. Universidade Estadual de Maringá (UEM). Maringá, Paraná, Brasil. ORCID: 0000-0002-7597-784X

\section{Maria Aparecida Salci}

Doutora em Enfermagem. Universidade Estadual de Maringá (UEM). Maringá, Paraná, Brasil. ORCID: 0000-0002-6386-1962

\section{INTRODUÇÃO}

A s doenças crônicas não transmissíveis (DCNT) representam uma crise global para a saúde pública, sendo o tabaco um dos principais fatores de risco para seu desenvolvimento ${ }^{(1)}$. O consumo de tabaco é responsável pela morte de 7 milhões de pessoas todos os anos, caracterizando uma epidemia glo$\mathrm{bal}^{(2)}$. Caso a tendência atual de consumo continue, no ano de 2030 haverá cerca de 8 milhões de óbitos decorrentes do uso de tabaco, sendo que $80 \%$ destes ocorrerão em países de baixa e média renda, como é o caso do Brasil ${ }^{(3)}$.

No cenário brasileiro, estima-se que 428 pessoas morrem diariamente em consequência do tabagismo, e $12,6 \%$ de todas as mortes registradas no país estão relacionadas ao consumo de tabaco. Ademais, cerca de 156.216 mortes poderiam ser evitadas anualmente com a cessação tabágica $^{(1)}$. Assim, no país, as ações individuais e coletivas com enfoque no tratamento e prevenção do tabagismo ficam sob a responsabilidade da Atenção Primária à Saúde (APS), regulamentada como lócus principal do cuidado a pessoa tabagista, por suas características de promotora do cuidado integral e longitudinal, consi- derado componentes necessários para o manejo e controle do tabagismo de forma contínua ${ }^{(5)}$ baseados nos princípios e diretrizes que compõem o Programa Nacional de Controle do Tabagismo (PNCT) ${ }^{(1,4)}$.

O PNCT busca reduzir o dano individual, social e ambiental dos produtos derivados do tabaco, objetivando reduzir o número de fumantes e consequentemente a morbimortalidade. Por meio de ações educativas, de comunicação e de Atenção à Saúde, o PNCT estabelece que a abordagem e o tratamento devem ser realizados prioritariamente nas Unidades Básicas de Saúde (UBS), devido sua capacidade de descentralização e capilaridade ${ }^{(4)}$. Nesse contexto, a Equipe de Saúde da Família (EqSF) é uma estratégia primordial de Atenção à Saúde e propõe-se a reorganizar a APS no Brasil, por oferecer a reorientação do processo de trabalho com maior potencialidade de ampliação e resolução, que possa impactar na situação de saúde dos indivíduos ${ }^{(5)}$.

Destacam-se como foco das ações a serem desenvolvidas pelo PNCT no âmbito da APS: reduzir a iniciação do consumo de tabaco; proteger a população contra os riscos do tabagismo passivo; reduzir a aceitação social do tabagismo; aumentar o acesso ao tratamento do fumante; contro- lar e monitorar os produtos derivados do tabaco; e monitorar as tendências de consumo/estratégias da indústria ${ }^{(4)}$.

Assim, o tratamento de tabagismo no Brasil é desenvolvido priorizando a técnica do aconselhamento estruturado/abordagem intensiva em formato de grupos de cessação tabágica. Esta técnica busca compreender os motivos que levam o indivíduo a fumar, e utiliza estratégias para que haja mudanças de crenças e comportamentos, visando não só a cessação do tabagismo, mas também a prevenção de recaídas a longo prazo. Em casos específicos podem ser utilizados medicamentos que sirvam de apoio a essa abordagem ${ }^{(4-6)}$.

Diante desse contexto, emergiu a seguinte questão de pesquisa: "Quais os desfechos do tratamento para a cessação tabágica ofertado por um município de médio porte do Estado do Paraná?". Para responder essa questão, o presente estudo objetivou analisar os desfechos do tratamento para a cessação tabágica oferecido pelas UBS, inseridas no Programa de Controle do Tabagismo (PCT), do município de Maringá/Pr.

\section{MÉTOdO}

Trata-se de um estudo documental, descritivo e de abordagem quantitativa 


\section{artigo}

que analisou os desfechos do tratamento para a cessação tabágica oferecido pelas UBS inseridas no PCT do município de Maringá/Pr, no período de 2015 a 2019. A escolha deste período justifica-se pela falta de dados consistentes dos anos anteriores para sustentar a análise do estudo.

O município de Maringá é considerado de médio porte e está localizado ao Noroeste do Estado do Paraná, com uma população estimada em 430.157 habitantes ${ }^{(7)}$. Entre 2015 a 2017 contava com 33 UBS e 68 EqSF, e a partir de 2018 houve expansão do serviço com abertura de mais uma UBS e seis novas EqSF, totalizando

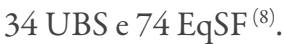

A coleta de dados se deu no período de março a junho de 2019 por meio de documentos oficiais da Secretaria Municipal de Saúde de Maringá e relatórios mensais e/ou anuais desenvolvidos pelas UBS do município que ofertaram grupos de apoio à cessação tabágica. Adotou-se como critérios de inclusão as UBS que ofertaram tratamento para o tabagismo no período da análise, e foram excluídos os dados que continham informações incompletas.

Os desfechos do tratamento para a cessação tabágica eleitos para análise nesse estudo foram: quantitativo de grupos realizados; taxa de iniciação do tratamento; taxa de conclusão; abandono das sessões; cessação tabágica ao término do tratamento e utilização da terapia medicamentosa.

Os resultados foram organizados e tabulados em planilha eletrônica no Software Microsoft Excel 2016, e foram analisados por estatística descritiva e inferencial.
Foi aplicado o teste qui-quadrado de Pearson com auxílio do ambiente estatístico Epinfo versão 7.0, no qual elaborou-se uma tabela de contingência com formato de $2 \times 2$. O nível de significância fixado foi de $\alpha=5 \%(p<0,05)$, assim, para que houvesse correlação positiva, o valor encontrado de $\mathrm{p}$ deveria ser menor que o nível de significância.

Esse estudo possui apreciação ética pela Comissão Permanente de Avaliação de Projetos - Portaria nº 009/2017/ SAÚDE e pelo Comitê Permanente de Ética em Pesquisa com Seres Humanos (COPEP) sob Certificado de Apresentação de Apreciação Ética (CAAE) $n^{\circ}$ 57222016.1.0000.0104 e parecer $\mathrm{n}^{\circ}$ 2.278.656.

\section{RESULTADOS}

No período de 2015 a 2019, o município de Maringá/Pr assistiu um total de 2.339 usuários em seus grupos de apoio a cessação tabágica nas UBS inseridas no PCT. Deste total, a taxa de conclusão do tratamento foi de 55,7\%, abandono $44,3 \%$, cessação do consumo $30 \%$ e uso de terapia medicamentosa $68,8 \%$ (Tabela 1 ).

No ano de 2015 foi observado uma maior taxa de iniciação ao tratamento da cessação tabágica (31,3\%). Ainda, 65,5\% concluíram o tratamento, 59,7\% cessaram o consumo de tabaco até a última sessão e $73,1 \%$ utilizaram algum medicamento como apoio para cessação. Com relação a taxa de abandono, cerca de $34,5 \%$ não concluíram as sessões (Tabela 1).
Entretanto, tais resultados não se mantiveram constantes, e nos anos seguintes foram verificadas quedas nas taxas de iniciação e, consequentemente, conclusão do tratamento. Em 2016, 64\% dos usuários concluíram as quatro sessões, e 63,9\% cessaram o uso de tabaco. Neste mesmo ano, $72,6 \%$ utilizaram o apoio medicamentoso, e $36 \%$ dos participantes abandonaram o tratamento (Tabela 1).

Em relação ao ano de 2017 também houve queda no quantitativo de pessoas que iniciaram o tratamento e aumento da taxa de abandono, na qual $46,1 \%$ abandonaram o grupo e $25,4 \%$ cessaram o consumo do tabaco. Neste ano, a taxa de usuários que utilizaram medicação também manteve-se alta, com 77,4\% (Tabela 1).

Nos anos de 2018 e 2019 o quantitativo de usuários que iniciaram tratamento para o tabagismo manteve-se praticamente estável, porém a taxa de cessação reduziu para $12,2 \%$ e a taxa de abandono elevou-se para $64,4 \%$. Consequentemente, a taxa de uso de medicação também diminuiu, sendo que $46,9 \%$ dos usuários que iniciaram o grupo utilizaram algum tipo de medicação como suporte para a cessação tabágica (Tabela 1 ).

Com relação ao desenvolvimento dos grupos de apoio a cessação tabágica pelas UBS de Maringá, observou-se que a oferta ao longo dos anos apresentou oscilações e declínio. Os anos de 2015 e 2016 foi o período com maior número de atividades realizadas, com um total de 30 UBS ofertando pelo menos um grupo durante o ano, prevalecendo aquelas que ofertaram três e um grupo anualmente. Nos anos seguintes, o

Tabela 1. Distribuição dos usuários pelo Programa de Controle do Tabagismo segundo desfecho do tratamento à cessação tabágica, entre 2015 a 2019. Maringá/Pr, 2021.

\begin{tabular}{|c|c|c|c|c|c|c|}
\hline \multirow{2}{*}{ DESFECHO DO TRATAMENTO } & 2015 & 2016 & 2017 & 2018 & 2019 & TOTAL \\
\hline & $\mathbf{N}(\%)$ & $\mathbf{N}(\%)$ & $\mathbf{N}(\%)$ & $\mathbf{N}(\%)$ & $\mathbf{N}(\%)$ & $\mathbf{N}(\%)$ \\
\hline Iniciação & $733(31,3)$ & $511(21,8)$ & $492(21)$ & $300(12,8)$ & $303(12,9)$ & 2339(100) \\
\hline Conclusão & $480(65,5)$ & $327(64)$ & $265(53,9)$ & $123(41)$ & $108(35,6)$ & $1303(55,7)$ \\
\hline Abandono & $253(34,5)$ & $184(36)$ & $227(46,1)$ & $177(59)$ & $195(64,4)$ & $1036(44,3)$ \\
\hline Cessação do consumo & $287(59,7)$ & $209(63,9)$ & $125(47,2)$ & $46(37,3)$ & $37(12,2)$ & $704(30,0)$ \\
\hline Uso de Terapia Medicamentosa & $536(73,1)$ & $371(72,6)$ & $381(77,4)$ & $179(59,6)$ & $142(46,9)$ & $1609(68,8)$ \\
\hline UBS que realizaram grupos & 30 & 30 & 23 & 18 & 16 & -- \\
\hline
\end{tabular}


quantitativo de UBS que realizaram grupos diminuiu, sendo 2019 o ano com a menor número de grupos ofertados no município. Neste mesmo ano, 16 UBS realizaram grupos de apoio a cessação tabágica, com a maioria realizando dois grupos no período, conforme apresentado na Tabela 2.

Observa-se que a maioria das UBS ofertaram apenas um grupo anualmente, $e$ o total de UBS que não realizaram grupos, mais que triplicou no período. Ao analisar e correlacionar os extremos de participação dos usuários no Programa, nos anos de 2015 e 2019, foi possível observar o nível de significância (Tabela 3). O número de usuários que concluíram o tratamento e cessaram o consumo de tabaco no ano de 2019 foi inferior quando comparado ao ano de 2015, assim como o total de usuários que iniciaram o tratamento e utilizaram algum tipo de medicação. Porém, ao observar o nível de significância, àqueles usuários que iniciaram o tratamento tiveram uma correlação significativa com a conclusão e com a cessação tabágica ao término das sessões do tratamento.

\section{DISCUSSÃO}

Este estudo analisou os desfechos do tratamento à cessação tabágica oferecido pelas UBS, inseridas em um PCT, do município de Maringá/Pr no período de 2015 a 2019. Ao analisar os desfechos, identificou-se que o tratamento ofertado à população pela APS baseava-se na execução de grupos de apoio a cessação tabágica com periodicidade trimestral e conduzidos por uma equipe de profissionais de saúde capacitados.

Reconhecendo que o tabagismo apresenta um padrão evolutivo semelhante às DCNT, podendo apresentar também períodos de remissões e recidivas, o MS atualizou as diretrizes da Rede de Atenção à Saúde das Pessoas com Doenças Crônicas, definindo que a identificação e o acolhimento à pessoa tabagista devem ser realizados em todos os pontos de atenção do SUS, prioritariamente nos serviços da APS ${ }^{(9)}$.

A APS foi reconhecida como a principal porta de entrada dos usuários aos serviços de saúde ofertados pelo SUS, sendo defini- do que a adesão das UBS a oferta do tratamento do tabagismo deve ser realizada pelo Programa Nacional de Melhoria do Acesso e da Qualidade da Atenção Básica (PMA$\mathrm{Q}-\mathrm{AB})$, que tem como objetivo induzir a ampliação do acesso e a melhoria da qualidade dos serviços, garantindo um padrão que permite maior transparência e efetividade das ações da APS em todo o país ${ }^{(9)}$.

A abordagem ao usuário que deseja cessar o uso do tabaco em formato de reunião grupal mostra-se como uma estratégia importante para o auxílio no processo de cessação. Esses grupos possuem uma característica de acompanhamento coletivo, com a realização de encontros periódicos que possibilitam a troca de experiências entre os participantes, permitindo a criação de vínculo entre profissionais e usuários e acompanhamentos posteriores após o término das reuniões em grupo, seguindo a proposta do Protocolo Clínico e Diretrizes Terapêuticas do Tabagismo $(2020)^{(10,6)}$.

Os grupos de cessação tabágica, seguindo os princípios do aconselhamento estruturado/abordagem intensiva, são re-

Tabela 2. Grupos de apoio à cessação tabágica desenvolvidos pelas Unidades Básicas de Saúde, por trimestre, entre 2015 a 2019. Maringá/Pr, 2021.

\begin{tabular}{lcccccc}
\multicolumn{1}{c}{ GRUPOS POR TRIMESTRE } & $\mathbf{2 0 1 5}$ & $\mathbf{2 0 1 6}$ & $\mathbf{2 0 1 7}$ & $\mathbf{2 0 1 8 ^ { * }}$ & $\mathbf{2 0 1 9 *}$ & \multicolumn{2}{c}{ TOTAL DE GRUPOS } \\
& $\mathbf{N ~ ( \% )}$ & $\mathbf{N}(\%)$ & $\mathbf{N ~ ( \% )}$ & $\mathbf{N}(\%)$ & $\mathbf{N}(\%)$ & $\mathbf{N}(\%)$ \\
Quatro & $\mathbf{7 ( 2 1 , 2 )}$ & $\mathbf{7 ( 2 1 , 2 )}$ & $6(18,2)$ & - & - & 20 \\
Três & $8(24,2)$ & $8(24,2)$ & $4(12,1)$ & $4(11,7)$ & $5(14,7)$ & 29 \\
Dois & $7(21,2)$ & $7(21,2)$ & $7(21,2)$ & $4(11,7)$ & $6(17,7)$ & 31 \\
Um & $8(24,2)$ & $8(24,2)$ & $6(18,2)$ & $10(29,5)$ & $5(14,7)$ & 37 \\
Sem & $3(9,2)$ & $3(9,2)$ & $10(30,3)$ & $16(47,1)$ & $18(52,9)$ & 50 \\
UBS com Grupos & 30 & 30 & 26 & 18 & 16 & -- \\
Fonte: dados da pesquisa, 2021. *Total de 34 UBS & & & & & &
\end{tabular}

Tabela 3. Correlação da participação dos usuários nos grupos de tabagismo nos anos de 2015 e 2019. Brasil, 2020.

\begin{tabular}{lccccc}
\multicolumn{1}{c}{ ANo } & \multicolumn{2}{c}{2015} & & 2019 & P \\
& N & $\%$ & N & $\%$ & \\
Iniciaram o tratamento & 733 & 303 & & & \\
Concluíram & 480 & 65,5 & 108 & 35,6 & 0,001 \\
Cessaram consumo & 287 & 59,7 & 37 & 12,2 & 0,001 \\
Utilizaram medicação & 536 & 73,1 & 172 & 46,9 & 0,07 \\
Fonte: dados da pesquisa, 2020. & & & & &
\end{tabular}

alizados em formato de reuniões grupais e devem ser compostos de pelo menos quatro sessões iniciais, realizadas semanalmente. O PCT recomenda ainda as sessões de manutenção, que devem ocorrer após a conclusão das sessões semanais. Inicialmente, estas devem acontecer quinzenalmente, com pelo menos duas sessões; e uma sessão mensal para prevenção da recaída até completar um ano do início do tratamento ${ }^{(4,6)}$. 
A Secretaria Municipal de Saúde oferece capacitações periódicas aos profissionais de saúde de acordo com os protocolos estabelecidos pelo MS, abordando o contexto do tabagismo, o que incluem desde a abordagem ao usuário tabagista, a sintomatologia e as metodologias preconizadas para o tratamento e o desenvolvimento dos grupos ${ }^{(4)}$.

No ano de 2015, três UBS do município ainda não possuíam profissionais de saúde capacitados para a execução destes grupos. Já nos anos seguintes, todas contavam com pelo menos um profissional de saúde capacitado por equipe para desenvolver as ações de tratamento, totalizando 151 profissionais aptos a desenvolver as ações do PCT junto à população, conforme recomendado pelo MS ${ }^{(6)}$.

Para garantir uma melhor assistência a esses usuários, o MS tornou obrigatória a capacitação de profissionais de saúde que atuam em grupos de cessação tabágica com treinamentos abordando aspectos históricos, preventivos e farmacológicos relacionados ao tabagismo, contextualizando o conhecimento sobre o tabaco e a política de saúde, prevenção e exposição tabágica, e ainda detalha a abordagem à pessoa tabagista e o papel do profissional de saúde no PCT ${ }^{(11)}$.

Outro aspecto observado nesse estudo foi o declínio em relação a oferta e procura de tratamento no município. Nota-se que o número de UBS que não realizaram nenhum grupo durante os anos analisados aumentou, e a assiduidade dos usuários ao longo das sessões diminuiu. A frequência no grupo correlaciona-se positivamente com o sucesso da cessação tabágica. O abandono aos grupos de tabagismo esteve presente em todos os anos analisados, e o número de participantes que abandonaram o tratamento antes do término de todas as sessões foi relativamente alto.

Outro estudo realizado também no município de Maringá/PR no ano de 2018, pontuou alguns aspectos relacionados à falta de adesão aos grupos de cessação tabágica, como a dificuldade de locomoção até a UBS $(73,43 \%)$, a falta de informação sobre o programa $(26,56 \%)$ e a incompatibilidade com a rotina ou falta de tempo $(17,18 \%)^{(12)}$.
A falta de adesão dos usuários ao tratamento também mostrou-se evidente em outro estudo que avaliou o desempenho de um PCT em uma UBS no município de São Luís/Maranhão no período de 2017. Observou que apesar do grande investimento do município em estratégias farmacológicas e não-farmacológicas para tratamento do tabagismo, o Programa se desenvolveu de maneira tímida, não havendo por parte da população, o devido reconhecimento sobre as ações de combate ao tabagismo oferecidas pelo SUS ${ }^{(13)}$.

Nesse sentido, uma

abordagem mais

criativa e dinâmica

a ser adotada nos

grupos poderia

atender à necessidade

dos participantes,

somados a uma

abordagem

focada nos fatores

desencadeantes do

desejo de fumar,

como a ansiedade,

a depressão, o

estresse e os hábitos

alimentares.
As intervenções terapêuticas não-farmacológicas para a cessação do tabagismo são consideradas estratégia primordial para auxílio no processo de cessação tabágica, visto que exerce uma relação custo-benefício efetiva, permitindo uma assistência simultânea à múltiplas pessoas, fazendo com que o indivíduo se sinta acolhido e consiga partilhar experiências e desafios ${ }^{(12)}$.

As abordagens utilizadas inicialmente envolvem avaliações referentes às comorbidades, história tabágica e grau de dependência de nicotina avaliado pelo teste de Fagerström. Após a entrevista inicial, o usuário é inserido em grupos de terapia cognitivo-comportamental (TCC), além de terapia medicamentosa (adesivos, goma de mascar e comprimidos) aos usuários com alto nível de dependência química à nicotina ${ }^{(4)}$.

A utilização da TCC como única estratégia, também parece não favorecer a continuidade do tratamento para a cessação tabágica ${ }^{(11)}$, o que reforça a ideia de readequação do grupo à realidade da população, adaptando a terapia a ser utilizada, visando uma maior taxa de adesão e, consequentemente, abandono do consumo do tabaco. Em uma revisão Cochrane, identificou-se que intervenções que combinem TCC com apoio medicamentoso aumentam o sucesso da cessação ao tabagismo quando comparado a intervenções isoladas ${ }^{(14)}$.

Nesse sentido, uma abordagem mais criativa e dinâmica a ser adotada nos grupos poderia atender à necessidade dos participantes, somados a uma abordagem focada nos fatores desencadeantes do desejo de fumar, como a ansiedade, a depressão, o estresse e os hábitos alimentares. Tais fatores poderiam garantir maior sucesso na participação dos usuários às atividades do Programa e na cessação do hábito de fumar ${ }^{(12)}$.

Com relação ao uso de medicação como auxílio ao tratamento, observou-se uma alta incidência em todos os anos analisados. Sabe-se que o tratamento medicamentoso aumenta significativamente as chances de as pessoas tabagistas alcançarem a cessação, porém, desempenha um papel auxiliar no acompanhamento do indivíduo, contribuindo para o alívio e o controle dos sinais e sintomas das crises de abstinência ${ }^{(4)}$. 
Um estudo sobre os fatores motivacionais para a redução ou cessação do tabagismo e dificuldades enfrentadas neste período, refuta que os sintomas de abstinência são barreiras encontradas pelas pessoas que desejam cessar o consumo do tabaco, reforçando que o uso da terapia farmacológica, como suporte para a cessação tabágica, é primordial para o sucesso do tratamento, principalmente quando associada às intervenções comportamentais ${ }^{(14)}$.

Destaca-se que o número de pessoas que fizeram uso de algum tipo de medicamento é relativamente maior ao número de participantes que concluíram o tratamento e alcançaram a cessação tabágica. Esta realidade aponta que as pessoas iniciam o tratamento, retiram o medicamento e em seguida o abandonam.

A partir do estudo realizado, observou-se uma queda no número de usuários que concluíram o tratamento e cessaram o consumo de tabaco no ano de 2018 quando comparado ao ano de 2015, o que reforça a necessidade de fomentar a formação de novos grupos, bem como a utilização de abordagens terapêuticas mais eficazes.

A limitação do estudo apresenta-se no fato de os dados retratarem apenas a realidade de um município do estado do Paraná, o que impossibilita a generalização dos resultados obtidos para outras realidades do país. Nesse sentido, sugere-se a realização de novos estudos para a discussão dessa temática, se propondo a avaliar os desfechos do tratamento do tabagismo, buscando compreender como o tratamento ofertado para a cessação tabágica tem impactado na vida dos usuários que o procuram por meio do PCT.

\section{CONCLUSÃO}

Analisar os desfechos do tratamento para a cessação tabágica oferecido pelas UBS inseridas em um PCT municipal do Estado do Paraná permitiu identificar que o tratamento era centralizado na oferta de grupos de apoio a cessação tabágica conduzidos por profissionais de saúde capacitados. Apesar dos investimentos na capacitação destes profissionais, nota-se uma queda com relação aos números relacionados a cessação tabágica ao término dos grupos e à oferta deste tratamento pelas UBS, com um aumento expressivo no quantitativo de Unidades que não desenvolveram nenhum grupo anualmente. Somado a isto, observa-se uma diminuição de usuários que iniciaram tratamento para o tabagismo no município.

Desta forma, recomenda-se investimentos dos gestores e dos profissionais de saúde da APS na melhoria deste tratamento, considerando a necessidade emergente a assistência à população tabagista, visto as consequências que o hábito de fumar acarreta aos usuários e ao sistema de saúde à longo prazo.

\section{REFERÊNCIAS}

1. INSTITUTO NACIONAL DO CÂNCER (INCA). Programa Nacional de Controle de Tabagismo. Brasília, DF. 2020. Disponível em: https://www.inca.gov.br/tabagismo.

2. WHO Report on the Global Tobacco Epidemic, 2019: Offer help to quit tobacco use. [s.I: s.n.].

3. WHO. WHO Report on the global tobacco epidemic, 2017: Monitoring tobacco use and prevention policies. [s.l: s.n.].

4. Estratégias para o cuidado da pessoa com doença crônica: o cuidado da pessoa tabagista. Brasília: Ministério da Saúde, 2015.

5. BRASIL. Ministério da Saúde. Portaria n².436, de 21 de setembro de 2017. Aprova a Política Nacional de Atenção Básica, estabelecendo a revisão de diretrizes para a organização da Atenção Básica, no âmbito do Sistema Único de Saúde (SUS). Brasília. 2017. Disponivel em: http://www.foa.unesp.br/home/ pos/ppgops/portaria-n-2436.pdf.

6. Protocolo Clínico e Diretrizes Terapêuticas do Tabagismo. Brasília: Ministério da Saúde, 2020.

7. INSTITUTO BRASILEIRO DE GEOGRAFIA E ESTATÍSTICA (IBGE). IBGE Cidades. Disponivel em: < https://cidades.ibge.gov.br/brasil/pr/maringa/panorama>. Acesso em: 04 fev. 2021.

8. Brasil, Ministério da Saúde. Banco de dados do Sistema Único de Saúde-DATASUS, Cadastro Nacional de Estabelecimentos de Atenção à Saúde - CNES Fluxo de Clientela. Disponivel em: < https:// http://cnes2.datasus.gov.br>. Acesso em: 04 fev. 2021.
9. BRASIL. Ministério da Saúde. Portaria n 571, de 05 de abril de 2013. Atualiza as diretrizes de cuidado à pessoa tabagista no âmbito da Rede de Atenção à Saúde das Pessoas com Doenças Crônicas do Sistema Único de Saúde (SUS) e dá outras providências. Brasília. 2013. Disponível em: http://bvsms.saude.gov.br/ bvs/saudelegis/gm/2013/prt0571_05_04_2013.html.

10. Fanshawe TR, Halliwell W, Lindson N, Aveyard P, Livingstone-Banks J, Hartmann-Boyce J. Tobacco cessation interventions for young people. Cochrane Database Syst Rev. 2017; 11(11):CD003289. doi: 10.1002/14651858.CD003289.pub6.

11. Brasil. Ministério da Saúde. Portaria no 1035, de 31 de maio de 2004. Amplia o acesso à abordagem e tratamento do tabagismo para a rede de atenção básica e de média complexidade do SUS. Brasília. 2004.

12. Pereira AAC, Gritsch LJ, Passos MS, Furtado MD. Adesão ao grupo de cessação entre tabagistas de unidade básica de saúde. Cogitare Enfermagem. 2018; 23(3). Doi: http://dx.doi. org/10.5380/ce.v23i3.55096

13. Ribeiro DBC. Avaliação do Programa Nacional de Controle do Tabagismo em São Luís. [Dissertação]. Coimbra. Escola Superior de Tecnologia da Saúde de Coimbra, Escola Superior de Educação de Coimbra; 2018. 68 p.

14. Pereira MO, Assis BCS, Gomes NMR, Alves AR, Reinaldo AMS, Beinner MA. Motivação e dificuldades para reduzir ou cessar o uso de tabaco. Rev. Bras. Enferm. 2020; 73(1): e20180188. Doi: http://dx.doi.org/10.1590/0034-7167-2018-0188. 